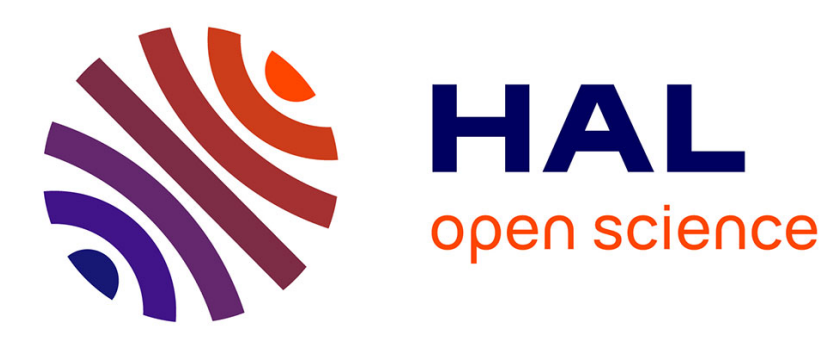

\title{
Coexistence in a fluctuating environment by the effect of relative nonlinearity: A minimal model
}

András Szilágyi, Géza Meszéna

\section{To cite this version:}

András Szilágyi, Géza Meszéna. Coexistence in a fluctuating environment by the effect of relative nonlinearity: A minimal model. Journal of Theoretical Biology, 2010, 267 (4), pp.502. 10.1016/j.jtbi.2010.09.020 . hal-00637812

\section{HAL Id: hal-00637812 \\ https://hal.science/hal-00637812}

Submitted on 3 Nov 2011

HAL is a multi-disciplinary open access archive for the deposit and dissemination of scientific research documents, whether they are published or not. The documents may come from teaching and research institutions in France or abroad, or from public or private research centers.
L'archive ouverte pluridisciplinaire HAL, est destinée au dépôt et à la diffusion de documents scientifiques de niveau recherche, publiés ou non, émanant des établissements d'enseignement et de recherche français ou étrangers, des laboratoires publics ou privés. 


\section{Author's Accepted Manuscript}

Coexistence in a fluctuating environment by the effect of relative nonlinearity: A minimal model

András Szilágyi, Géza Meszéna

PII:

S0022-5193(10)00494-7

DOI: doi:10.1016/j.jtbi.2010.09.020

Reference: YJTBI 6160

To appear in: $\quad$ Journal of Theoretical Biology

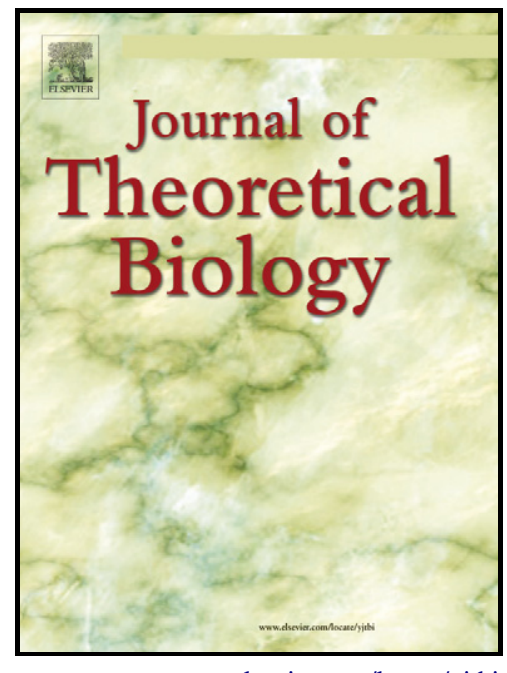

www.elsevier.com/locate/yjtbi

Received date: 14 January 2010

Revised date: $\quad 14$ September 2010

Accepted date: $\quad 15$ September 2010

Cite this article as: András Szilágyi and Géza Meszéna, Coexistence in a fluctuating environment by the effect of relative nonlinearity: A minimal model, Journal of Theoretical Biology, doi:10.1016/j.jtbi.2010.09.020

This is a PDF file of an unedited manuscript that has been accepted for publication. As a service to our customers we are providing this early version of the manuscript. The manuscript will undergo copyediting, typesetting, and review of the resulting galley proof before it is published in its final citable form. Please note that during the production process errors may be discovered which could affect the content, and all legal disclaimers that apply to the journal pertain. 


\title{
Coexistence in a fluctuating environment by the effect of relative nonlinearity: a minimal model
}

\author{
András Szilágyi ${ }^{1, *}$ \\ Department of Plant Taxonomy and Ecology, Eötvös University \\ Pázmány Péter sétány $1 C, H-1117$ Budapest, Hungary \\ Géza Meszéna \\ Department of Biological Physics, Eötvös University \\ Pázmány Péter sétány $1 \mathrm{~A}, \mathrm{H}-1117$ Budapest, Hungary
}

\begin{abstract}
The minimal model of the "relative nonlinearity" type fluctuation-maintained coexistence is investigated. The competing populations are affected by an environmental white noise. With quadratic density dependence, the long-term growth rates of the populations are determined by the average and the variance of the (fluctuating) total density. At most two species can coexist on these two "regulating" variables; competitive exclusion would ensue in a constant environment. A numerical study of the expected time until extinction of any of the two species reveals that the criterion of mutual invasibility predicts the parameter-range of long-term coexistence correctly in the limit of zero extinction threshold. However, any extinction threshold consistent with a realistic population size will allow only short-term coexistence. Therefore, our simulations question the biological relevance of mutual invasibility, as a sufficient condition of coexistence, for large density fluctuations. We calculate the average and the variance of the fluctuating density of the coexisting populations analytically via the moment-closure approximation; the results are reasonably close to the simulated behavior. Based on this treatment, robustness of coexistence is studied in the limit of infinite population size. We interpret the results of this analysis in the context of necessity of niche segregation with respect to the regulating variables using a framework theory published earlier.
\end{abstract}

Keywords: niche, limiting similarity, stochastic population dynamics

-

\footnotetext{
${ }^{*}$ Corresponding author

Email addresses: and.szilagyi@gmail.com (András Szilágyi), geza.meszena@elte.hu (Géza Meszéna)

${ }^{1}$ phone: +36-1-372-2795, fax: +36-1-372-2757
}

Preprint submitted to Elsevier

September 17, 2010 


\section{Introduction}

The role of environmental fluctuations in maintenance of species diversity is one of the most frequently investigated topics in theoretical ecology.

The classical reference point is equilibrium coexistence theory. The principle of competitive exclusion (Gause 1934, Hardin 1960) states that only the most successful species can survive in a given niche. That is, coexistence requires ecological (niche) segregation; limiting similarity of the coexisting species is expected. The principle is often stated in a narrower form: the number of populations coexisting at a fixed point attractor cannot exceed the number of resources (Armstrong 1976). More generally, instead of the resources, one should count all of the environmental variables involved in the feedback loop of population regulation (Levin 1970). In line with e.g. Krebs (2001), p. 288 and Meszéna et al. (2006), we will refer to these variables as regulating factors/variables. Following the lead of May (1973), Vandermeer (1975) and Abrams (1983), Meszéna et al. (2006) proved in a model-independent way that increasing similarity between the populations makes their coexistence less likely, i.e. more sensitive to the perturbation of external parameters. For robust coexistence, the populations should differ in their relationship to the regulating variables - i.e. in their impact on, and sensitivity towards, these variables. Therefore, a population's (differential) impacts and sensitivities constitute the proper model-independent generalization of the resource utilization function as a descriptor of the population's niche (Meszéna et al. 2006). Szabó and Meszéna (2006), Barabás and Meszéna (2009) studied the loss of robustness with disappearing niche segregation in the Lotka-Volterra model. Szilágyi and Meszéna (2009a,b) applied the theory to spatial niche segregation in a heterogeneous environment. Here we investigate usefulness of this approach in fluctuating environment.

Hutchinson (1961) had already questioned the universal validity of the equilibrium coexistence theory and raised the question whether the high diversity of plankton communities could be explained by either the fluctuating nature of the environment or the internal dynamics of the system. Indeed, Armstrong (1976) demonstrated that the number of coexisting species can exceed the number of resources even in a constant environment if the population dynamical attractor is not a fixed point. During the ' 80 s it has become a widely held opinion that the Hutchinson-MacArthur-era overestimated the relevance of competitive exclusion compared with nonequilibrium processes (Huston 1979, 1994, Begon et al. 1996). On the other hand, Abrams (1983), Chesson (1991), Chesson and Huntly (1997), Chesson et al. (2004) stressed that fluctuations do not alleviate the need for ecological segregation. Even in a fluctuating environment the species having the largest long-term rate of increase (calculated via proper averaging) will outcompete the others. Therefore, competitive exclusion remains the default behavior.

According to Chesson (2000b), coexistence must be stabilized by a negative feedback that gives a boost to any of the populations that become rare. In a constant environment, niche segregation of the classical kind provides this feedback: the rare species will have an abundant supply of resources. For fluc- 
tuating environments Chesson (1994) enlists two additional mechanisms that are specifically related to fluctuations: the "storage effect" and the "effect of relative nonlinearity". The first one is essentially a temporal niche-segregation, made possible by the fluctuations (Christiansen and Fenchel 1977, p. 69, Amarasekare 2003), while the second one means that the statistical moments of the fluctuating regulating variables emerge as additional regulating variables via averaging of non-linear dependencies (cf. Levins 1979, Kisdi and Meszéna 1993). Chesson (2003) provided estimations for the strength of the two mechanisms, allowing to asses their relative importance. The spatiality-related coexistence mechanisms, described in Chesson (2000a), Amarasekare (2003), Amarasekare et al. (2004), Szilágyi and Meszéna (2009a,b), are outside of our interest here.

Turelli (1978) formulated the necessity of a stabilizing feedback as a sufficient condition for coexistence: if either of the two populations is able to grow when rare enough, then they are able to coexist. This criterion of mutual invasibility is widely used to simplify theoretical investigations of coexistence.

The principal goal of the present paper is to study the relation between the theory of fluctuation-mediated coexistence and the classical notion of niche, as formulated by Meszéna et al. (2006). While we aim for the general picture, here we use the simplest model that we could conceive to demonstrate the principal connection between equilibrium and nonequilibrium ecology (see Parvinen and Meszéna 2009 for a more realistic model of disturbance-generated coexistence in the same vein). For this purpose we use a slightly generalized version of the relative nonlinearity-type coexistence model by Kisdi and Meszéna (1993). There are only two regulating variables in this model, the long-term average and the variance of the total density. Therefore, it is ensured that at most two species can coexist.

Because of the simplicity of the model, an approximate analytical treatment is possible via the method of moment closure. We will compare these approximate results with numerical simulations. The analytic solution allows us to calculate the populations' impact and sensitivity vectors, which are the descriptors of the population's niche in the framework theory. This way we will be in the position to consider the robustness of coexistence in terms of segregation of the niches. After introducing our model in Section 2, we describe the basic analytic and numerical results on coexistence in Sections 3 and 4 . Section 5 discusses the moment closure approximation. Finally, Section 6 provides the niche-theoretical analysis.

\section{Building the model}

Since we wish to study principal issues, we look for a model of fluctuationmediated coexistence as close to analytical tractability as possible. Continuous time is preferred, as discrete-time population dynamics tends to exhibit more complicated behavior. The simplest source of fluctuations is the uncorrelated external noise. We expect competitive exclusion to operate in the absence of fluctuations. This is ensured if the dependence on the total density is the only regulating feedback in the model (density-dependent selection, MacArthur 
1962). If density-dependence were linear, fluctuations would not affect the behavior (Chesson and Huntly 1997). Quadratic dependence is the minimalist route to the effect of relative nonlinearity.

Therefore, the fluctuations will be driven by a Gaussian uncorrelated (white) noise $\xi(t)$ with zero mean $(\bar{\xi}=0$, the overline denotes time averaging). Its autocorrelation function can be written as

$$
\overline{\xi\left(t_{1}\right) \xi\left(t_{2}\right)}=\delta\left(t_{1}-t_{2}\right)
$$

where $\delta\left(t_{1}-t_{2}\right)$ is the "Dirac-delta function", that is zero for $t_{1} \neq t_{2}$, but has an integral of 1 , specifying the normalization.

Then, our model is defined by the stochastic differential equation (SDE)

$$
\frac{\mathrm{d}}{\mathrm{d} t} n_{i}(t)=\overbrace{\left[\sigma_{i} \xi(t)-a_{i}\left(n(t)-K_{i}\right)-b_{i}\left(n(t)-K_{i}\right)^{2}\right]}^{r_{i}(t)} n_{i}(t)
$$

where $n_{i}(t)$ is the density of Species $i$ at time $t, n(t)=\sum_{i} n_{i}(t)$ is the total density. The expression in the square bracket, denoted by $r_{i}(t)$, is the instantaneous growth rate of Species $i . K_{i}$ is the carrying capacity of the $i$ th Species, i.e. the equilibrium density without noise; $a_{i}, b_{i}$ and $\sigma_{i}$ are positive constants. The ratio $b_{i} / a_{i}$ characterizes the nonlinearity of population regulation, while the noise-intensity coefficient $\sigma_{i}$ specifies the dependence of the population on the external fluctuations. To avoid complications arising from an Allee-effect, we want the deterministic part of the growth rate to decrease monotonously with increasing density. To this end, the parameter values are chosen to satisfy the inequality

$$
\frac{a_{i}}{2 b_{i}}>K_{i}
$$

We use the Stratonovich interpretation of the SDE (see e.g. May 1973, p. 204; Braumann 1999, 2007 and Appendix for the different interpretations of SDEs). The intuitive advantage of this choice is that the time-average of the instantaneous growth rate should be zero for any species that survives for a long period of time, if time averaging (denoted by overline) is defined via Stratonovich integration (Braumann 2007). In our model this time-average reads as

$$
\bar{r}_{i}=-a_{i}\left(\bar{n}-K_{i}\right)-b_{i} \overline{\left(n-K_{i}\right)^{2}}+\sigma_{i} \bar{\xi}=-a_{i}\left(\bar{n}-K_{i}\right)-b_{i}\left(\bar{n}-K_{i}\right)^{2}-b_{i} V
$$

where we denote the variance of the total density $n$ by $V=\overline{n^{2}}-\bar{n}^{2}$. Observe the detrimental effect of the fluctuations on the long-term growth rate through the quadratic term. The "equilibrium" condition is $\bar{r}_{i}=0$.

Without fluctuations, $V=0$. Then $\bar{r}_{i}$ becomes zero when the time-averaged total density $\bar{n}$ reaches the carrying capacity $K_{i}$. By the well-known argument of MacArthur (1962), competition is won by the species with the highest carrying capacity in this case.

In the presence of external fluctuations the densities will also fluctuate, implying $V>0$. The average growth rates are receiving feedback through two 
"regulating" variables: $\bar{n}$ and $V$. Each surviving population provides one equilibrium equation $\left(\bar{r}_{i}=0\right.$ for Species $\left.i\right)$ for these variables. The generic solution exists only if the number of equations does not exceed the number of unknowns. That is, coexistence of two, but not more, species is allowed in this model.

Since this bound on diversity is clear from the onset of our investigations, it is instructive to write down the dynamics (2) again just for two species:

$$
\begin{aligned}
& \frac{\mathrm{d}}{\mathrm{d} t} n_{1}(t)=\left[\sigma_{1} \xi(t)-a_{1}\left(n(t)-K_{1}\right)-b_{1}\left(n(t)-K_{1}\right)^{2}\right] n_{1}(t), \\
& \frac{\mathrm{d}}{\mathrm{d} t} n_{2}(t)=\left[\underline{\sigma_{2} \xi(t)}-a_{2}\left(n(t)-K_{2}\right)-\underline{b_{2}\left(n(t)-K_{2}\right)^{2}}\right] n_{2}(t) .
\end{aligned}
$$

The special case $b_{2}=\sigma_{2}=0$, i.e. when the underlined terms are deleted, will be referred to as the simplified model, first published by Kisdi and Meszéna (1993). Since we are interested in limiting similarity here, we need the full model that will allow the two species to become equivalent when their parameters are equal. However, as the simplified model has a fewer number of parameters, it is more convenient for the study of the coexistence of two species that are distinct.

\section{Elementary analytic results}

In this Section we summarize the analytical results that do not rely on the moment closure approximation; the latter will be considered in Section 5. The calculations presented here are direct consequences of the equilibrium conditions. While they are valid for arbitrarily large fluctuations, we will often use the approximation of small fluctuations to keep the formulae transparent.

\subsection{Single species}

First we consider the long-term equilibrium of a single species. The equilibrium condition can be written as

$$
\bar{r}=-a(\hat{n}-K)-b(\hat{n}-K)^{2}-b \hat{V}=0
$$

(cf. Eq. (4)), where $\hat{n}$ and $\hat{V}$ denote the average and the variance of the population density, respectively, for the case of a lone population. In a constant environment $\hat{V}=0$, implying $\hat{n}=K$. As density variance has a negative effect on the growth rate and there is no Allee effect (monotonicity condition (3)), the average density must decrease with increasing density fluctuation.

For small fluctuations we expect a small $\hat{V}$ implies a small value of $\hat{n}-K$ characterizing the departure of the average density from the constant-environment value. In this limit we can neglect the second order small term in Eq. (7) leads to the relation

$$
\hat{n}-K=-\frac{b}{a} \hat{V}
$$

That is, the difference $\hat{n}-K$ is proportional to $\hat{V}$. In the small fluctuation approximation we will neglect the terms beyond the linear order in either quantity. 
Note that the terms, like the second one in Eq. (7), will always be negligible for small fluctuations. Therefore, the essential role of the nonlinear terms $-b_{i}\left(n_{i}-K_{i}\right)^{2}$ in dynamics $(7)$ is to implement a sensitivity towards the fluctuations on the density. The ratio $b / a$ that measures the nonlinearity of the density dependence also characterizes the sensitivity of the average density towards density fluctuations. In the case of linear density dependence $(b=0)$, fluctuations of the density average out; the average density will not be affected by the fluctuations.

\subsection{Mutual invasion}

The condition of mutual invasibility of two species reads

$$
\begin{aligned}
& \varrho_{1}=-a_{1}\left(\hat{n}_{2}-K_{1}\right)-b_{1}\left(\hat{n}_{2}-K_{1}\right)^{2}-b_{1} \hat{V}_{2}>0, \\
& \varrho_{2}=-a_{2}\left(\hat{n}_{1}-K_{2}\right)-b_{2}\left(\hat{n}_{1}-K_{2}\right)^{2}-b_{2} \hat{V}_{1}>0 .
\end{aligned}
$$

Here $\varrho_{i}$ denotes the "boundary" growth rate of Species $i$, i.e. its growth rate, when it is rare and the other species fluctuates in a stationary manner. While these conditions are exact, the quantities $\hat{n}_{i}$ and $\hat{V}_{i}$ cannot be calculated analytically without the moment closure approximation of Section 5 .

A more concise condition can be derived for the simplified model with $b_{2}=$ $\sigma_{2}=0$. In this case Species 2 is affected by the fluctuations only through its interaction with Species 1. When alone, Species 2 assumes an equilibrium density determined by the condition $\bar{r}_{2}=-a_{2}\left(\hat{n}_{2}-K_{2}\right)=0$, implying $\hat{n}_{2}=K_{2}$. Then the boundary growth rate of Species 1 is

$$
\varrho_{1}=-a_{1}\left(K_{2}-K_{1}\right)-b_{1}\left(K_{2}-K_{1}\right)^{2} .
$$

It is positive iff

$$
K_{2}<K_{1}
$$

where inequality (3) was used. On the other hand, Species 2 has a positive boundary growth rate against the established population of Species 1 iff

$$
\varrho_{2}=-a_{2}\left(\hat{n}_{1}-K_{2}\right)>0 .
$$

The combination of Eqs. (10) and (12) provides the necessary and sufficient condition for mutually invasibility as

$$
\hat{n}_{1}<K_{2}<K_{1}
$$

(cf. Kisdi and Meszéna 1993). There is no exact analytic way to determine $\hat{n}_{1}$; it has to come either from simulations or from moment closure approximation applied to a single species. 


\subsection{Advantage of rarity}

Advantage of rarity (negative frequency dependence in the terminology of population genetics) is an essential component of coexistence. Following Chesson (2003), we measure it by the quantity

$$
\varrho=\frac{\varrho_{1}}{a_{1}}+\frac{\varrho_{2}}{a_{2}} .
$$

Mutual invasibility implies $\varrho>0$, but not vice versa. Obviously, this would be true for any linear combination of $\varrho_{1}$ and $\varrho_{2}$ with positive coefficients. We justify the normalization by the $a_{i}$ s via an argument modified from Chesson (2009). In case of linear density dependence $\varrho_{1} / a_{1}=K_{2}-K_{1}$ and $\varrho_{2} / a_{2}=K_{1}-K_{2}$. Therefore, the chosen normalization arranges that $\varrho=0$ for the reference case of linearity, when relative nonlinearity cannot operate. The essential issue behind this normalization is that the quantity $r_{1} / a_{1}-r_{2} / a_{2}$ is independent of the total density $n$ in the linear case.

We will use the approximation of small fluctuations. Straightforward calculation leads to

$$
\varrho=\left[\frac{b_{1}}{a_{1}}-\frac{b_{2}}{a_{2}}+2 \frac{b_{1} b_{2}}{a_{1} a_{2}} \Delta K\right]\left(\hat{V}_{1}-\hat{V}_{2}\right)-\left[\frac{b_{1}}{a_{1}}+\frac{b_{2}}{a_{2}}\right](\Delta K)^{2}>0,
$$

where the notation

$$
\Delta K=K_{1}-K_{2}
$$

was introduced. For $\Delta K=0$ it simplifies to the following transparent formula

$$
\left(\frac{b_{1}}{a_{1}}-\frac{b_{2}}{a_{2}}\right)\left(\hat{V}_{1}-\hat{V}_{2}\right)>0 .
$$

Condition (17) represents the coexistence-stabilizing mechanism and corresponds to the last term of Eq. (7) in Chesson (2000b), see also Eq. (68) in Chesson (1994) and the explanation thereafter. It has an intuitive meaning pointing to a close analogy between fluctuation-dependent and fluctuation-independent mechanisms to maintain coexistence. As mentioned above, the ratio $b_{i} / a_{i}$ measures the nonlinearity of density-dependence of Species $i$; their difference in the first factor is the "relative" nonlinearity (Chesson 1994, 2000b). Phrasing it differently, the first factor represents the difference between the species in their dependence on the two regulating variables, the average $\bar{n}$ and the variance $V$ of the total density (cf. Section 6). On the other hand, the second term is the difference between the species in their strength of making the total population size fluctuates. For coexistence, the species must differ in both respects. Moreover, they must differ in these aspects in the same way. The species that makes $n$ more fluctuating must be the one that is more sensitive to the fluctuations of $n$. The species that affects the regulating variable $V$ must be the one that is also more sensitive toward $V$. Like in constant-environment resource partitioning, this arrangement ensures rare advantage and stabilizes coexistence. We will revisit this expectation in Section 6.2 in a different way. 
Obviously, conditions (15-17) provide only the necessary, and not the sufficient conditions for mutual invasibility. They represent the conditions for having a tendency for rare advantage. If the parameter choices (esp. of $\Delta K$ ) are such that one species has a large advantage, then it is possible that the inferior species will be unable to invade despite its advantage gained from rarity.

\subsection{Coexistence}

Assume now that the two species coexist. Then their long-term growth rates satisfy the equilibrium equations

$$
\begin{aligned}
& \bar{r}_{1}=-a_{1}\left(\bar{n}-K_{1}\right)-b_{1}\left(\bar{n}-K_{1}\right)^{2}-b_{1} V=0, \\
& \bar{r}_{2}=-a_{2}\left(\bar{n}-K_{2}\right)-b_{2}\left(\bar{n}-K_{2}\right)^{2}-b_{2} V=0 .
\end{aligned}
$$

These (quadratic) conditions determine the equilibrium values of the regulating variables $\bar{n}$ and $V$ via a $4^{\text {th }}$ order algebraic equation. We write down the solution for small fluctuations, i.e. in linear order in $V$. For $V=0$ coexistence is possible only if $K_{1}=K_{2}$; the solution is characterized by $\bar{n}=K_{1}=K_{2}$. Therefore, we expect that small $V$ implies $K_{1} \approx K_{2}$ and then $\bar{n} \approx K_{1} \approx K_{2}$. Therefore in the small fluctuation limit - as in Eq. (8) - the second terms in both equations are of second order small; neglecting them leads to

$$
\bar{n}=\frac{a_{1} b_{2} K_{1}-a_{2} b_{1} K_{2}}{a_{1} b_{2}-a_{2} b_{1}}
$$

and

$$
V=\frac{K_{1}-K_{2}}{\frac{b_{1}}{a_{1}}-\frac{b_{2}}{a_{2}}} .
$$

Note the consistence of these formulae with the assumptions we made: smallness of $V$ implies smallness of $K_{1}-K_{2}$ as a condition for coexistence. The analogue of Eq. (8),

$$
\bar{n}-K_{i}=-\frac{b_{i}}{a_{i}} V
$$

remains valid for the two-species case by the same argument from which it was derived in Section 3.1.

For the simplified model,

$$
\begin{gathered}
\bar{n}=K_{2} \\
V=\frac{a_{1}}{b_{1}} \Delta K
\end{gathered}
$$

applies. 


\section{Coexistence: simulation results}

For simulation purposes, the continuous dynamics (2) is discretized as

$$
n_{i, t+\Delta t}-n_{i, t}=\left[-a_{i}\left(n_{t}-K_{i}\right)-b_{i}\left(n_{t}-K_{i}\right)^{2}+\frac{1}{2} \sigma_{i}^{2}\right] n_{i, t} \Delta t+\sigma_{i} n_{i, t} \sqrt{\Delta t} \xi_{t},
$$

where $n_{i, t}$ and $n_{t}$ denote the density of Species $i$ and the total density, respectively, at time $t$. The noise $\xi_{t}$ is an i.i.d. process of variance 1 for time steps of length $\Delta t$. The scaling factor $\sqrt{\Delta t}$ in the random term ensures that the normalization (1) is obeyed in the $\Delta t \rightarrow 0$ limit. The last term in the square bracket is the correction characteristic of Stratonovich integration (see Appendix; Sethi and Lehoczky 1981, Stratonovich 1989, Braumann 2007).

A simulated time series of two coexisting species is plotted in Fig. 1 for a set of parameters satisfying the mutual invasibility condition. In line with the invasibility prediction, they coexist for an arbitrary long period of time. Nevertheless, one should note that the densities fluctuate by many orders of magnitude. The populations experience extremely low densities.

Excursions to low densities warrants introduction of an extinction threshold. This way extinction of one of the species becomes a probability 1 event; the possibility of coexistence for infinite time is lost. Then the appropriate question is the expected length of coexistence.

Fig. 2 presents the average time until one of the densities goes below the threshold as a function of $K_{2}$ for different threshold values. Since the simplified model is used, condition (13) provides the parameter range for mutual invasibility, depicted by vertical dashed lines. Note that the plateau on the top of the curves is an artifact of terminating the simulations at $T_{\max }=10^{7}$ time steps. Fig. 3 shows the average length of coexistence as a function of the extinction threshold. The two curves represent two parameter sets that do and do not allow for mutual invasibility. In the first case the length of coexistence - after a transient period - increases linearly as a function of the inverse of the extinction threshold. In contrast, the expected time until extinction increases very slowly and remains essentially bounded when the condition of mutual invasibility is not met.

The presented results confirm the prediction of the mutual invasibility criterion unequivocally for sufficiently low extinction thresholds. The expected length of coexistence is bounded only by the simulation time $T_{\max }$ where the condition is met, but falls off by orders of magnitude outside the range of mutual invasibility. On the other hand, one should note that the "sufficiently low" extinction threshold is unrealistically low from biological point of view. The extinction threshold $10^{-20}$, i.e. population size $10^{20}$ is required for coexistence length $10^{6}$ even in case of mutual invasibility. For realistic population sizes the period of coexistence is very short.

The extreme level of density fluctuations are presumably related to the lowfrequency components of the white noise. Therefore, introduction of a lowfrequency cutoff of the power spectrum would make our model a good candidate 
for describing real species coexistence. Note that bounded periodic drive would result in bounded oscillation of the densities; therefore they would never become exponentially small.

However, for the rest of the current model, we remain interested in the analytic study of the white-noise model in the hope for insights that remain valid for more parameter-rich models.

\section{Moment closure approximation}

The equilibrium equations (18) are insufficient to fully determine the statistics of the population fluctuations. When a population is considered alone (Section 3.1), this condition establishes a relation between the average and the variance of the density, but does not allow us to calculate these quantities separately. For two populations, the average and variance of the sum of the densities are obtainable (Section 3.4), but not the statistics of the two populations separately. For more detailed analytic results the moment closure approximation will be used: we assume small fluctuations and neglect the third and higher statistical moments of the fluctuating variables.

The long-term average of the time-derivative of any quantity, which remains bounded, should be zero. Applying this principle to the logarithmic density $\ln n_{i}$ (time derivative of which is the growth rate $r_{i}$ ) leads to the equilibrium condition $\overline{r_{i}}=0$. We have exploited this condition already in Section 3.1. We need an additional relation to proceed further. For this purpose here we consider the average of the time derivative of $n_{i}$, instead of $\ln n_{i}$ :

$$
\overline{\frac{\mathrm{d}}{\mathrm{d} t} n_{i}(t)}=\overline{r_{i} n_{i}}=\overline{\left[\sigma_{i} \xi(t)-a_{i}\left(n(t)-K_{i}\right)-b_{i}\left(n(t)-K_{i}\right)^{2}\right] n_{i}}=0 .
$$

We introduce the deviation $m_{i}=n_{i}-\bar{n}$ and use the notation $m=m_{1}+m_{2}=$ $n-\bar{n}$. Note that

$$
\overline{m(t)}=\overline{m_{i}(t)}=0
$$

and

$$
\overline{m^{2}}=\overline{m_{1} m}+\overline{m_{1} m}=V .
$$

Moreover, we will use the relation

$$
\overline{m_{i} \xi}=\frac{\sigma_{i}}{2} \bar{n}_{i}
$$

which is proven in the Appendix.

We define the moment closure approximation by neglecting the moments of $m$ beyond $\overline{m^{2}}$. It is consistent with the small fluctuation approximation according to the relation (27). When the relationships (26-28) are taken into account, the moment closure version of condition (25) reads as

$$
\frac{\sigma_{i}^{2}}{2} \bar{n}_{i}-a_{i} \bar{n}_{i}\left(\bar{n}-K_{i}\right)-a_{i} \overline{m_{i} m}-b_{i} \bar{n}_{i}\left(\bar{n}-K_{i}\right)^{2}-b_{i} \bar{n}_{i} V-2 b_{i} \overline{m_{i} m}\left(\bar{n}-K_{i}\right)=0 .
$$


Now we substitute Eq. (21) into the expressions $\left(\bar{n}-K_{i}\right)$ and according to the small fluctuation limit after neglecting all second order terms in $V$ we arrive at the equality

$$
\frac{\sigma_{i}^{2}}{2} \bar{n}_{i}=a_{i}\left(1-\frac{2 b_{i}}{a_{i}} V\right) \overline{m_{i} m} .
$$

Neglecting the term $V \overline{m_{i} m}$, which is of order $\overline{m^{4}}$, leads to

$$
\frac{\sigma_{i}^{2}}{2 a_{i}} \bar{n}_{i}=\overline{m_{i} m} .
$$

With summation of this formula for $i=1,2$ one arrives to

$$
V=\frac{\sigma_{1}^{2}}{2 a_{1}} \bar{n}_{1}+\frac{\sigma_{2}^{2}}{2 a_{2}} \bar{n}_{2}=v_{1} \bar{n}_{1}+v_{2} \bar{n}_{2},
$$

where the notation

$$
v_{i}=\frac{\sigma_{i}^{2}}{2 a_{i}}
$$

was introduced. Note the intuitive meaning of $v_{i}$ : it is the per capita contribution of Species $i$ to the variance of the total population size. Equation (32), together with the trivial relation

$$
\bar{n}=\bar{n}_{1}+\bar{n}_{2}
$$

establish the connection between the average densities of the two species and the two regulating variables. One can solve the system of equations $(32,34)$ for the average densities:

$$
\begin{gathered}
\bar{n}_{1}=\frac{V-v_{2} \bar{n}}{v_{1}-v_{2}}, \\
\bar{n}_{2}=\frac{-V+v_{1} \bar{n}}{v_{1}-v_{2}} .
\end{gathered}
$$

These results, together with equations (19-20), provide all the interesting quantities as a function of the model parameters.

For the simplified model, substitution of $b_{2}=\sigma_{2}=0$ and Eqs. (22-23) results in

$$
\begin{gathered}
\bar{n}_{1}=\frac{2 a_{1}^{2}}{b_{1} \sigma_{1}^{2}} \Delta K=\frac{\hat{n}_{1}}{K_{1}-\hat{n}_{1}} \Delta K, \\
\bar{n}_{2}=K_{2}-\frac{2 a_{1}^{2}}{b_{1} \sigma_{1}^{2}} \Delta K=K_{2}-\frac{\hat{n}_{1}}{K_{1}-\hat{n}_{1}} \Delta K .
\end{gathered}
$$

For one species a similar moment closure calculation leads to the relations

$$
\hat{n}=\left(1-\frac{\sigma^{2} b}{2 a^{2}+\sigma^{2} b}\right) K=\frac{2 a^{2} K}{2 a^{2}+\sigma^{2} b},
$$

and

$$
\hat{V}=\frac{\sigma^{2} a K}{2 a^{2}+\sigma^{2} b} .
$$


Fig. 4 presents the comparison of this one-species result with numerical simulations. The coincidence of the predicted and simulated average densities is remarkable; the fluctuations need not to be extremely small for this. The moment closure method turns out to be reliable as long as the noise intensity parameter $\sigma$ is not larger than the parameters $a$ and $b$ in the regulation terms. Observe that the equilibrium density is a monotonically decreasing function of the strength of the external fluctuations. With very high fluctuations the average density can be made arbitrary small, provided that the extinction threshold is small enough.

The analogous comparison between moment closure (Eq. (36)) and simulation is presented for two species in Fig. 5. The departure of the moment closure prediction from the simulated values is larger than in case of a single species. Still, the agreement is reasonable. The moment closure approximation seems to capture the essential behavior of the system. Observe that the deviation is the same in magnitude, but opposite in direction, for the two species. That is, the total density is still provided accurately; the moment closure method is less precise in predicting the ratio of the two densities. This situation is understandable in the light of high sensitivity of the relative densities for the fluctuations.

\section{Niche segregation and robustness of coexistence}

Based on the analytic results above, in this section we reinvestigate the model in the context of the theory of limiting similarity by Meszéna et al. (2006). First we briefly reiterate how it works in a constant environment; then we consider the current model.

\subsection{Reference theory: constant environment}

Here we summarize the theory of limiting similarity by Meszéna et al. (2006). It is a model-independent implementation of the proposal by Abrams (1983) that limits to similarity must be considered in conjunction with the relative competitiveness of the coexisting species; see also Chesson (2000b) in the same vein. The key statement of Meszéna et al. is that species should differ in their way of regulation for robust coexistence; the more similar they are, the more narrow is the range of competitive parameters allowing for their coexistence. More specifically, one should consider the species' impact on, and sensitivity towards the regulating factors. Strength of competition between the populations is reduced and their coexistence is robust if they differ in both respects.

Write the dependence of the growth rate of the $i$ th species $(i=1,2, \ldots, L)$ on the densities $n_{1}, \ldots, n_{L}$, as

$$
r_{i}=r_{i}\left(\boldsymbol{I}\left(n_{1}, n_{2}, \ldots, n_{L}\right), \boldsymbol{E}\right) \quad(i=1,2, \ldots, L),
$$

where the vector $\boldsymbol{I}=\left(I_{1}, I_{2}, \ldots, I_{D}\right)$ collects the $D$ number of regulating variables; $\boldsymbol{E}$ denotes the vector of "external" variables that affect the population 
growth rates but are not affected by the population densities. Then, the elements of the competition matrix are

$$
\alpha_{i j}=-\frac{\partial r_{i}}{\partial n_{j}}=\sum_{k=1}^{D} S_{i k} C_{j k}=\boldsymbol{S}_{i} \cdot \boldsymbol{C}_{j},
$$

where the partials

$$
C_{i k}=\frac{\partial I_{k}}{\partial n_{i}}, \quad S_{i k}=-\frac{\partial r_{i}}{\partial I_{k}}
$$

measure the $i$ th species' impact on, and sensitivity towards, the $k$ th regulating factor, respectively. The vectors $\boldsymbol{C}_{i}=\left\{C_{i 1}, C_{i 2}, \ldots, C_{i D}\right\}$ and $\boldsymbol{S}_{i}=$ $\left\{S_{i 1}, S_{i 2}, \ldots, S_{i D}\right\}$ will be referred to as the impact and sensitivity niches of the $i$ th species. The sensitivity of the equilibrium population sizes towards the additional mortalities can be determined by implicit differentiation of the equilibrium equation $r_{i}=0$ :

$$
\frac{\partial n_{i}^{\mathrm{e}}}{\partial \boldsymbol{E}}=-\sum_{j=1}^{L}\left(\alpha^{-1}\right)_{i j} \frac{\partial r_{j}}{\partial \boldsymbol{E}}=-\frac{1}{J} \sum_{j=1}^{L} \operatorname{adj}(\alpha)_{i j} \frac{\partial r_{j}}{\partial \boldsymbol{E}},
$$

where $\operatorname{adj}(\alpha)$ denotes the adjunct of the community matrix. The determinant $J=\operatorname{det}\left(\alpha_{i j}\right)$ measures the strength of regulation at the community level. As it appears in the denominator, a weakly regulated community is sensitive towards the extra mortalities. Therefore a small $|J|$ implies that only a small range of the external parameters can be tolerated without extinction of one of the species. Robust coexistence requires large $|J|$. Note that if the sensitivity niches of two different species coincide, the corresponding two rows of matrix $\alpha$ are the same and $J=0$. A similar argument holds for the impact vectors and the columns of the matrix. Therefore, the requirement of large $|J|$ translates to the requirement of sufficiently different niche vectors.

\subsection{Impact and sensitivity niches in our system}

The sensitivity analysis in Section 6.1 is based on the assumption of equilibrium in an essential way. Its applicability for our model is not trivial.

A part of the framework is easy to translate. The growth rate $r_{i}$ of the constant environment model should be replaced by the averaged growth rate $\bar{r}_{i}$ because the "equilibrium" equation $\bar{r}_{i}=0$ holds even in our variable environment. As we discussed already, the averaged total density $\bar{n}$ and the variance of the total density $V$ play the role of regulating variables: these quantities determine the $\bar{r}_{i}$ s unequivocally. Therefore, the vector of regulating factors can be written as

$$
\boldsymbol{I}=\left(\begin{array}{c}
\bar{n} \\
V
\end{array}\right)
$$

while the sensitivity vectors are

$$
\boldsymbol{S}_{\boldsymbol{i}}=-\left(\begin{array}{c}
\frac{\partial \bar{r}_{i}}{\partial \overline{\bar{n}}_{i}} \\
\frac{\partial \bar{r}_{i}}{\partial V}
\end{array}\right)=\left(\begin{array}{c}
a_{i}+2 b_{i}\left(\bar{n}-K_{i}\right) \\
b_{i}
\end{array}\right) .
$$


Omitting $\bar{n}-K_{i} \sim V$ the formula simplifies to

$$
\boldsymbol{S}_{\boldsymbol{i}} \approx\left(\begin{array}{c}
a_{i} \\
b_{i}
\end{array}\right)
$$

In this sense one can say that the coefficients of the linear and the quadratic density dependence measure the sensitivity towards the average and the variance of the total population size, respectively. Note however, that here we neglect a term which is linear in $V$, because the leading term is independent of $V$.

The nontrivial part is the interpretation of the equilibrium density $n_{i}^{\mathrm{e}}$. The average density $\bar{n}_{i}$ is the obvious candidate. However, the average density of the species alone does not determine the regulating vectors. Therefore, the impact vectors cannot be defined, and the formalism cannot be applied without further considerations.

To apply the framework we have to restrict the possibilities of the stochastic dynamics to a two-parameter sub-family that can be parameterized by $\bar{n}_{1}$ and $\bar{n}_{2}$. We do this by picking up the carrying capacities $K_{1}$ and $K_{2}$ as competitive parameters (Abrams 1983), playing the role of the vector $\boldsymbol{E}$ above. All other model parameters are regarded as fixed; robustness of coexistence is considered with respect to changes of the carrying capacities. This way both the average densities $\bar{n}_{1}, \bar{n}_{2}$ and the regulating variables $\bar{n}, V$ become functions of the carrying capacities $K_{1}, K_{2}$. Inversion of these relationships leads to an unequivocal definition of the dependence of the regulating variables on the average densities. Note that a similar reduction of dimensionality was applied when population structure caused an analogous problem (Szilágyi and Meszéna 2009a,b).

Then the impact niche vector of Species $i$ is calculable from the relations (32) and (34) as

$$
\boldsymbol{C}_{\boldsymbol{i}}=\left(\begin{array}{c}
\frac{\partial \bar{n}}{\partial \bar{n}_{i}} \\
\frac{\partial V}{\partial \bar{n}_{i}}
\end{array}\right)=\left(\begin{array}{c}
1 \\
v_{i}
\end{array}\right) .
$$

From the vectors $\boldsymbol{S}_{i}$ and $\boldsymbol{C}_{j}$ we can obtain the competiton matrix:

$$
\alpha_{i j}=-\frac{\partial r_{i}}{\partial \bar{n}_{j}}=\boldsymbol{S}_{i} \cdot \boldsymbol{C}_{j}
$$

As explained in Section 6.1, the determinant of this matrix is the measure of the robustness we are looking for:

$$
\frac{\operatorname{det}(\boldsymbol{\alpha})}{a_{1} a_{2}}=\left(\frac{b_{1}}{a_{1}}-\frac{b_{2}}{a_{2}}+2 \frac{b_{1} b_{2}}{a_{1} a_{2}} \Delta K\right)\left(v_{1}-v_{2}\right),
$$

where Eqs. (44) and (45) were used.

Observe resemblance of this measure of regulatedness to the quantity $\varrho$ in the l.h.s. of the necessary condition (15) in Section 3.3 for coexistence. While invasion from rarity was considered in Section 3.3, here we studied the effect of a small perturbation. This difference in the approaches explains the discrepancies between the results. First, while the per capita fluctuation-producing effect of the species $\left(v_{1}\right.$ and $\left.v_{2}\right)$ appears here, the analogous quantities for the whole 
populations $\left(\hat{V}_{1}\right.$ and $\left.\hat{V}_{2}\right)$ were used in Section 3.3. Second, as here we considered robustness with respect to $\Delta K$, the dependence on $\Delta K$ is different from the one in Section 3.3.

These differences notwithstanding, the intuitive meaning of the robustness measure (47) is the same as of $\varrho$ in Section 3.3: the species must differ both in their fluctuation-maintaining effect and their sensitivities towards fluctuations. The differential approach here has the advantage of implementing the modelindependent connection between robustness and niche segregation (Meszéna et al. 2006). This formalism makes the analogy between fluctuation-related and fluctuation-unrelated coexistence, which we explained after Eq. (17), quantitative.

\subsection{Robustness of coexistence}

Here we demonstrate the loss of robustness of coexistence when the two species becomes similar. For this purpose the simplified model is rewritten as

$$
\begin{gathered}
\frac{\mathrm{d}}{\mathrm{d} t} n_{1}(t)=\left[\mu \sigma \xi(t)-a\left(n(t)-K_{1}\right)-\mu b\left(n(t)-K_{1}\right)^{2}\right] n_{1}(t), \\
\frac{\mathrm{d}}{\mathrm{d} t} n_{2}(t)=-a\left(n(t)-K_{2}\right) n_{2}(t)
\end{gathered}
$$

where $\mu$ is the parameter measuring dissimilarity. The two species are identical for $\mu=0$ and become dissimilar with increasing $\mu$. The robustness of coexistence is determined by varying the carrying capacities.

Fig. 6 plots the ratio of the parameter region $\left(K_{1}, K_{2}\right) \in[0,0.3] \times[0,0.3]$, which allows coexistence, as a function of $\mu$. Observe that robustness is lost gradually when the species become more and more similar. Coexistence becomes structurally unstable for $\mu=0$, when only the case $K_{1}=K_{2}$ supports coexistence.

This result, which is in line with theoretical expectations, is comparable with Fig. 6.4-6.6 of May (1973), or with Figs. 4-5 of Szilágyi and Meszéna (2009b).

\section{Discussion}

We aimed at studying the simplest possible model of fluctuation-mediated coexistence as deeply as possible in the context of a mathematical niche theory published earlier (Meszéna et al. 2006, Szilágyi and Meszéna 2009a,b, Parvinen and Meszéna 2009). In line with this approach, simplicity is measured by the number of regulating variables. As a single regulating factor cannot support coexistence, we constructed a model with two of them (see Kisdi and Meszéna 1993 for the original version). While all "strategic" (Czárán 1998, p. xii) model studies pay a price in terms of realism, our sacrifice turned out to be higher than usual. The coexistence, which is maintained in our model in the infinite population size limit, requires astonishingly large population sizes. Its practical irrelevance notwithstanding, our model provided a test-bed for developing moment-closure treatment of problems and developing niche analyses in 
line with our theoretical context. The model supported the idea that sufficient niche differentiation with respect to the way populations are regulated is the requirement for robust coexistence in an environment that displays stationary fluctuations - just like in a constant one.

Our wider context is classical coexistence theory. It was conceived in the framework of the Lotka-Volterra model and disregarded stochasticity. The resulting picture was simple and intuitive: coexistence was based on ecological niche differentiation leading to reduced interspecific competition and rare advantage (Case 2000, p. 368). However, as no clear lower bound of similarity was found (Abrams 1983), further model studies blurred this simplicity. Strength of competition and niche have become terms of unclear meaning, terms to be defined separately in every specific situation. The goal of Meszéna et al. (2006) was to reestablish the intuitive as well as the mathematical clarity of coexistence theory in a general, model-independent way. Competition coefficients were defined differentially; therefore they became independent of the assumption of linearity in the Lotka-Volterra model. Niche was defined as the species' differential impact on, and differential sensitivity towards the regulating variables, a generalization of the concept of the resource utilization function. Clear general connection between niche segregation and robustness of coexistence was established. It was asserted that complications like population structure and environmental fluctuations can be tackled within this framework via time-scaling arguments (Meszéna et al. 2006). The theory was extended by Szilágyi and Meszéna $(2009 \mathrm{a}, \mathrm{b})$ for structured populations in a constant environment. The goal of the current paper was to apply the same framework for the minimal model of fluctuation-maintained coexistence.

There are two distinct sources of stochasticity in population dynamics: environmental noise and demographic stochasticity (Case 2000, p. 30). While both of them are present in almost any real ecological situation, it is meaningful to consider the approximations under which either or both of them are negligible. Demographic stochasticity is the more difficult problem. Neglecting it is equivalent to assuming infinite population size, i.e. describing population dynamics in terms of continuous density variables. In this case, even if environmental noise is present, the dynamics is deterministic at least in the conditional sense, i.e. for a given realization of the environmental process. In contrast, finite population size leads to demographic stochasticity unavoidably. (Even local finiteness of a spatially extended infinite population results in local demographic stochasticity, see Oborny et al. 2005, 2007.) Analytic treatment of the stochastic dynamics of finite populations is mainly restricted to density-independent growth (cf. branching process theory, Haccou et al. 2005); but see Klebaner (2005), p. 366; Jagers (2010), Klebaner et al. (in press) for intriguing progress beyond this limitation.

In many situations environmental stochasticity maintains coexistence that would not be otherwise possible. In contrast, demographic stochasticity is invariably detrimental for coexistence, because it causes rare disadvantage and extinction. While analytic studies of fluctuation-maintained coexistence often assume infinite population size, numerical investigations can never completely 
disregard the consequences of finiteness. Accordingly, we discuss fluctuationrelated coexistence first for infinite populations then with considering finiteness.

In the limit of infinite population size it is self-evident that mutual invasion implies long-term coexistence; numerical experimentation with our model supported this principle unequivocally. It is the basis of the comprehensive theory of fluctuation-mediated coexistence by Chesson (1994) (see also Chesson 2009 for a very readable summary). Chesson's formalism and Meszéna et al. (2006) share the feature of being framework theories: both of them represent a common mathematical structure behind a class of models.

Chesson (1994) stresses the distinction between fluctuation-independent and fluctuation-dependent mechanisms for coexistence and concentrates on the latter. In particular, he studies emergence of coexistence-maintaining effects from the process of time averaging. Averaging implement a "scale transition" (Chesson 2009) between the original time scale and the long time scale of stationarity. His basic conclusion is that coexisting species should average the environmental process differently - otherwise the species with the highest average growth rate would win.

In contrast, the complementary approach by Meszéna et al. (2006) concentrates on the commonality of all kinds of coexistence-maintaining mechanisms (fluctuating, or not) on the time scale of stationarity. At this level the common cause for coexistence is segregation with respect to the "regulating" variables. Any fluctuation-dependent maintenance mechanism must be represented by proper regulating variables at the long time scale. On one hand, our model is consistent with Chesson's framework. Our formulas often directly correspond to his one. On the other hand, the current work is an initial exercise to apply the theory by Meszéna et al. (2006) for fluctuation-mediated coexistence.

The central issue of commonality of coexistence-maintaining mechanisms is loss of robustness of coexistence with increasing similarity of the species. In equilibrium resource competition the species, which depletes a specific resource more than its competitor, must depend on that specific resource more strongly to establish a coexistence-stabilizing rare advantage. Weak differentiation results in weak robustness against environmentally induced relative advantage/disadvantage between the species - a well known, but rarely stressed, phenomenon on the Lotka-Volterra model (Vandermeer 1975; May 1973, Fig. 6.4 on p. 158). In clear analogy, the relative nonlinearity mechanism for coexistence requires the species that causes the larger fluctuations of the total density to be more sensitive towards the fluctuations as well. If the populations become similar in either respect, then the populations must be fine tuned for coexistence. This is expressed by Eq. (68) in Chesson (1994) or Eq. (2) in Chesson (2009); the same issue is encountered in our Eqs. (17) and (47).

In this paper we turned the analogy between fluctuation-independent and -dependent mechanisms into an essential mathematical connection. We were able to determine the impact/sensitivity niche vectors for our model and demonstrate their expected connection to robustness of coexistence. For the analytic results we relied on the moment closure approximation, i.e. we assumed small fluctuations. However, the basic definitions, as well as of the underlying theory, 
are independent of these approximations; therefore, the principal connection we advocate is also exact. Note the similar conclusions in other models of fluctuation-mediated coexistence. Parvinen and Meszéna (2009) studied the case of repeated local catastrophes, while Barabás et al. (in prep.) investigated periodic environments.

Now we turn our attention to the unavoidable consequences of demographic stochasticity. Any population with bounded population size is destined to extinction with probability 1. Mutual invasibility has the tendency to increase the time-span of coexistence, but it cannot prevent eventual extinction. When demographic stochasticity is relatively small, it remains true that mutual invasibility (and the niche-segregation behind it) can maintain coexistence on the biologically relevant time-scale. However, as our example shows, the interaction between environmental and demographic stochasticity may lead to large density fluctuations that makes the diversity-stabilizing effect of mutual invasibility irrelevant. Therefore, we should caution against the uncritical use of mutual invasibility as a sufficient condition for coexistence, a point that was already made by Proulx and Day (2001). Johansson and Ripa (2006), Johansson et al. (2010) studied evolutionary consequences of finiteness, while Mágori et al. (2005), Habets et al. (2007) dealt with the coexistence-restricting effect of local demographic stochasticity.

The observed unreliability of mutual invasibility, as a predictor of coexistence, does not imply uselessness of the principle. As it is exact in the limit of infinite population size, it remains the correct tool to study whether environmental stochasticity has a coexistence-maintaining effect in a given ecological situation. However, one has to be aware that demographic stochasticity always has an opposite effect. This opposite effect can be negligible in some cases, can completely eliminate coexistence in others, or it may have an intermediate importance. It is entirely possible that our model will turn out to be extremely unfortunate in this respect. As the effect of relative nonlinearity is a weak force for coexistence, it requires large environmental fluctuations for robust coexistence, causing large density fluctuations. On the other hand, storage effect can be less vulnerable to demographic stochasticity: "storage" actually means buffering at rarity. The bottom line is that studies of mutual invasibility are useful to be complemented by investigating time-to-extinction with extinction threshold.

Within the confines of the current model, the extremely large density-fluctuations are presumably related to the low-frequency end of the white-noise spectrum of the environmental fluctuation: extremely low densities are consequences of unfavorable environmental conditions experienced for an extended period of time. Therefore we conjecture that the same model with a different noise spectrum, while complicates the analytic treatment and increases the number of parameters, will turn out to be a biologically more relevant description of fluctuation-mediated coexistence. 


\section{Acknowledgements}

We thank György Barabás, Carlos Braumann, Géza Györgyi, Éva Kisdi, Dina Lantos, Peter Jagers, Hans Metz, Liz Pásztor and Zoltán Rácz for discussions and two anonymous referees for useful comments on the original verision of the ms. This work was financed by OTKA grants K81628 and NK73047. 
Abrams, P. A., 1983. The theory of limiting similarity. Annual Review of Ecology and Systematics 14, 359-376.

Amarasekare, P., 2003. Competitive coexistence in spatially structured environments: a synthesis. Ecol. lett. 6, 1109-1122.

Amarasekare, P., Hoopes, M. F., Mouquet, N., Holyoak, M., 2004. Mechanism of coexistence in competitive metacommunities. Am. Nat. 164, 310-326.

Armstrong, R. A., 1976. Coexistence of species competing for shared resource. Theoretical Population Biology 9, 317-328.

Barabás, G., Meszéna, G., 2009. When the exception becomes the rule: the disappearance of limiting similarity in the Lotka-Volterra model. Journal of theoretical Biology 258, 89-94.

Begon, M., Harper, J. L., Townsend, C. R., 1996. Ecology. $3^{\text {rd }}$ ed. Blackwell Science Publisher, London.

Braumann, C. A., 1999. Applications of stochastic differential equations to population growth. In: Bainov, D. (Ed.), Proceedings of the Ninth International Colloquium on Differential Equations. VSP, Leiden, p. 47.

Braumann, C. A., 2007. Itô versus stratonovich calculus in random population growth. Mathematical Biosciences 206, 81-107.

Case, T. J., 2000. An Illustrated Guide to Theoretical Ecology. Oxford University Press.

Chesson, P., 1991. A need for niches? Trends in Ecology and Evolution 6, 26-28.

Chesson, P., 1994. Multispecies competition in variable environment. Theoretical Population Biology 45, 227-276.

Chesson, P., 2000a. General theory of competitive coexistence in spatiallyvarying environments. Theoretical Population Biology 58, 211-237.

Chesson, P., 2000b. Mechanism and maintenance of species diversity. Annual Review of Ecological Systems 31, 343-366.

Chesson, P., 2003. Quantifying and testing coexistence mechanisms arising from recruitment fluctuations. Theoretical Population Biology 64, 345-357.

Chesson, P., 2009. Scale transition theory with special reference to species coexistence in a variable environment. Journal of Biological Dynamics 3, 149-163.

Chesson, P., Gebauer, R. L. E., Schwinning, S., Huntly, N., Wiegand, K., Ernest, M. S. K., Sher, A., Novoplansky, A., Weltzin, J. F., 2004. Resource pulses, species interactions, and diversity maintenance in arid and semi-arid environments. Oecologia 141, 236-253. 
Chesson, P., Huntly, N., 1997. The roles of harsh and fluctuating conditions in the dynamics of ecological communities. American Naturalist 150, 519-533.

Christiansen, F. B., Fenchel, T. M., 1977. Theories of populations in biological communities. Springer-Verlag, Berlin.

Czárán, T., 1998. Spatiotemporal models of population and community dynamics. Population and Community Biology series. Chapman \& Hall, London.

Feldman, M. W., Roughgarden, J., 1975. A population's stationary distribution and chance of extinction in a stochastic environment with remarks on the theory of species packing. Theoretical Population Biology 7, 197.

Gause, G. F., 1934. The struggle for existence. Williams and Wilkins, Baltimore.

Habets, M. G., Czárán, T., Hoekstra, R. F., de Visser, J. A. G., 2007. Spatial structure inhibits the rate of invasion of beneficial mutations in asexual populations. Proceedings of the Royal Society B 274, 2139-2143.

Haccou, P., Jagers, P., Vatutin, V., 2005. Branching Processes: Variation, Growth and Extinction of Populations. Vol. 5 of Cambridge Studies in Adaptive Dynamics. Cambridge University Press, Cambridge.

Hardin, G., 1960. Competitive exclusion. Science 131, 1292-97.

Huston, M. A., 1979. A general hypothesis of species diversity. American Naturalist $113,81-101$.

Huston, M. A., 1994. Biological Diversity. The coexistence of species on changing landscapes. Cambridge University Press, Cambridge.

Hutchinson, G. E., 1961. The paradox of the plankton. Am. Nat. 45, 137-145.

Jagers, P., 2010. A plea for stochastic population dynamics. Journal of Mathematical Biology 60, 761-764.

Johansson, J., Ripa, J., 2006. Will sympatric speciation fail due to stochastic competitive exclusion? Am. Nat. 168, 572-578.

Johansson, J., Ripa, J., Kuckländer, N., 2010. The risk of competitive exclusion during evolutionary branching: Effects of resource variability, correlation and autocorrelation. Theoretical Population Biology 77, 95-104.

Kisdi, É., Meszéna, G., 1993. Density dependent life history evolution in fluctuating environment. In: Yoshimura, J., Clark, C. W. (Eds.), Adaptation in Stochastic Environments. Vol. 98 of Lecture Notes in Biomathematics. Springer-Verlag, pp. 26-62.

Klebaner, F. C., 2005. Introduction to stochastic calculus with applications. Imperial College Press. 
Klebaner, F. C., Sagitov, S., Vautin, V. A., Haccou, P., Jagers, P., in press. Stochasticity in the adaptive dynamics of evolution: the bare bones. Journal of Biological Dynamics.

Krebs, C. J., 2001. Ecology. The experimental analysis of distribution and abundance. Benjamin Cummings, San Francisco, California.

Levin, S. M., 1970. Community equlibria and stability, and an extension of the competitive exclusion principle. American Naturalist 104(939), 413-423.

Levins, R., 1979. Coexistence in a variable environment. American Naturalist $114,765-783$.

MacArthur, R. H., 1962. Some generalized theorems of natural selection. Proceedings of the National Academy of Sciences USA 48, 1893-97.

Mágori, K., Szabó, P., Mizera, F., Meszéna, G., 2005. Adaptive dynamics in cellular automaton: Role of spatiality in competition, coexistence and evolutionary branching. Evolutionary Ecology Research 7, 1-21.

May, R. M., 1973. Stability and Complexity in Model Ecosystems. Princeton University Press, Princeton.

Meszéna, G., Gyllenberg, M., Pásztor, L., Metz, J. A. J., 2006. Competitive exclusion and limiting similarity: a unified theory. Theoretical Population Biology 69, 68-87.

Oborny, B., Meszéna, G., Szabó, G., 2005. Dynamics of populations on the verge of extinction. Oikos 109, 291-296.

Oborny, B., Szabó, G., Meszéna, G., 2007. Survival of species in patchy landscapes: percolation in space and time. In: Storch, D., Marquet, P. A., Brown, J. H. (Eds.), Scaling biodiversity. Cambridge University Press, Cambridge.

Parvinen, K., Meszéna, G., 2009. Disturbance-generated niche-segregation in a structured metapopulation model. Evolutionary Ecology Research 11, 651666.

Proulx, S. R., Day, T., 2001. What can invasion analyses tell us about evolution under stochasticity in finite populations? Selection 2, 2-15.

Sethi, S. P., Lehoczky, J. P., 1981. A comparison of the Itô and the Stratonovich formulations of problems in finance. Journal of Economic Dynamics and Control 3, 343-356.

Smythe, J., Moss, F., McClintock, P. V. E., Clarkson, D., 1983. Itô versus stratonovich revisited. Physics Letters A 97(3).

Stratonovich, R. L., 1989. Some Markov methods in the theory of stochastic processes in nonlinear dynamical systems. In: Moss, F., McClintock, P. V. E. (Eds.), Noise in nonlinear dynamical systems. Volume 1: Theory of continuous Fokker-Planck systems. Cambridge University Press, Cambridge, UK. 
Szabó, P., Meszéna, G., 2006. Limiting similarity revisited. Oikos 112, 612-619.

Szilágyi, A., Meszéna, G., 2009a. Limiting similarity and niche theory for structured populations. Journal of theoretical Biology 258, 27-37.

Szilágyi, A., Meszéna, G., 2009b. Two-patch model of spatial niche segregation. Evolutionary Ecology 23, 187-205.

Turelli, M., 1978. Re-examination of stability in randomly varying versus deterministic environments with comments on stochastic theory of limiting similarity. Theoretical Population Biology 13, 244-267.

Vandermeer, J. H., 1975. Interspecific competition: A new approach to the classical theory. Science 188, 253-255. 

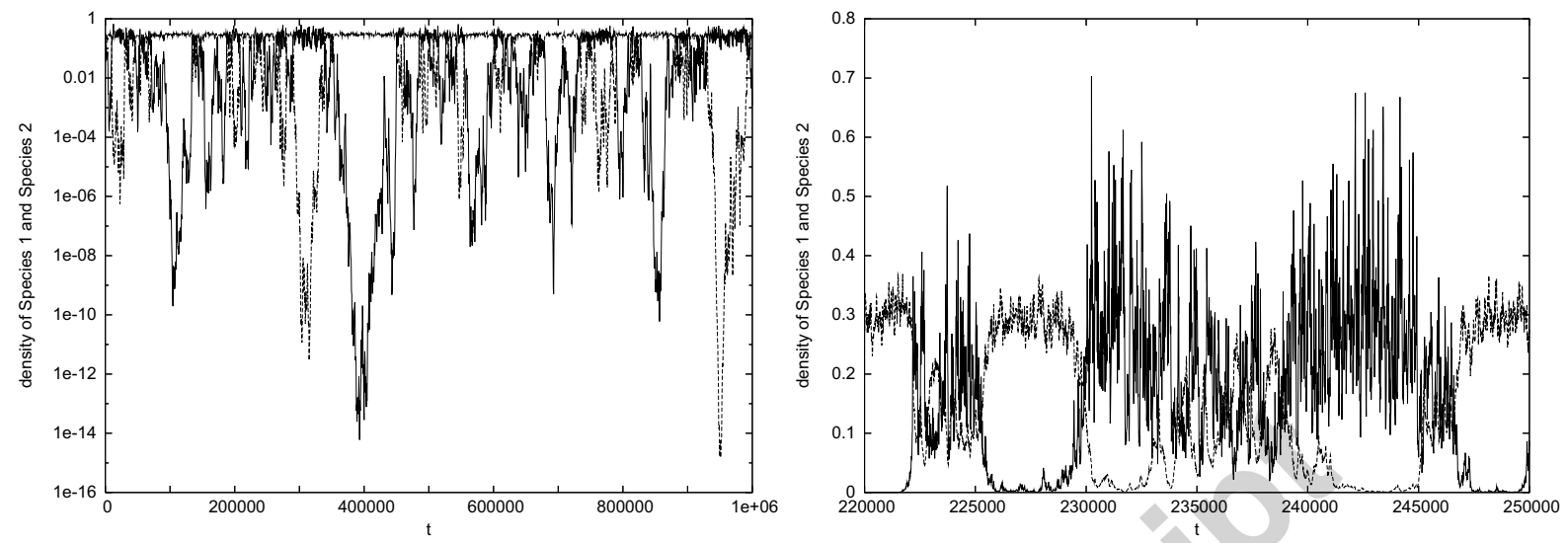

Figure 1: Time course of the densities of the two coexisting species. The left pane with logarithmic vertical scale demonstrates the regular occurrence of extremely low densities, incompatible with realistic population sizes. A part of the same curve is presented with linear scale on the right pane. Parameters: $a_{1}=a_{2}=b_{1}=0.1 ; b_{2}=0.02 ; \sigma_{1}=0.1 ; \sigma_{2}=0.05$; $K_{1}=0.3 ; K_{2}=0.298$. The same parameters are used for the rest of the Figures unless indicated otherwise. 

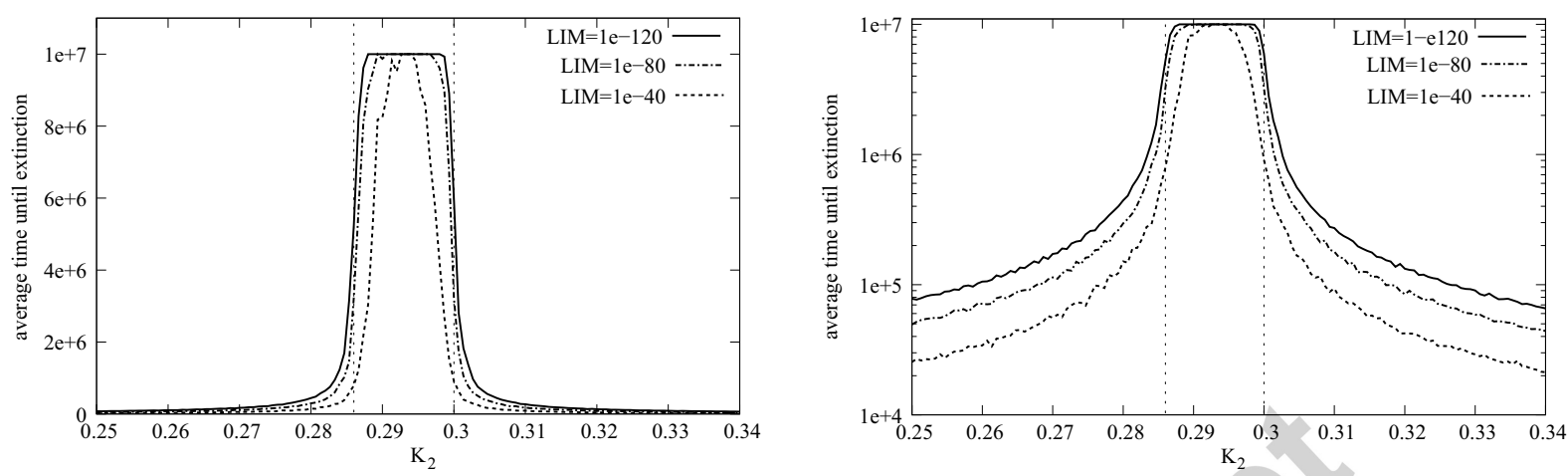

Figure 2: Expected time until extinction of one of the co-occurring species in the presence of an extinction threshold (LIM) as a function of the carrying capacity $K_{2}$ (simplified model, average of 50 runs). Vertical (time) scale is linear on the left plot and logarithmic on the right one. The simulations were terminated at time $T=10^{7}$ if no extinction occurred. The region of mutual invasibility $\left(\hat{n}_{1}, K_{1}\right)$ is shown by the vertical dashed lines. For extremely low extinction thresholds the parameter region of long-term coexistence coincides with that range. For more realistic thresholds, the possibility for coexistence is more restricted. Parameters are the same as in Fig. 1 except that now $b_{2}=\sigma_{2}=0$. This parameter choice is maintained in later figures of the simplified model when not indicated otherwise. The equilibrium density of Species 1, when alone, is measured to be $\hat{n}_{1}=0.286$. 


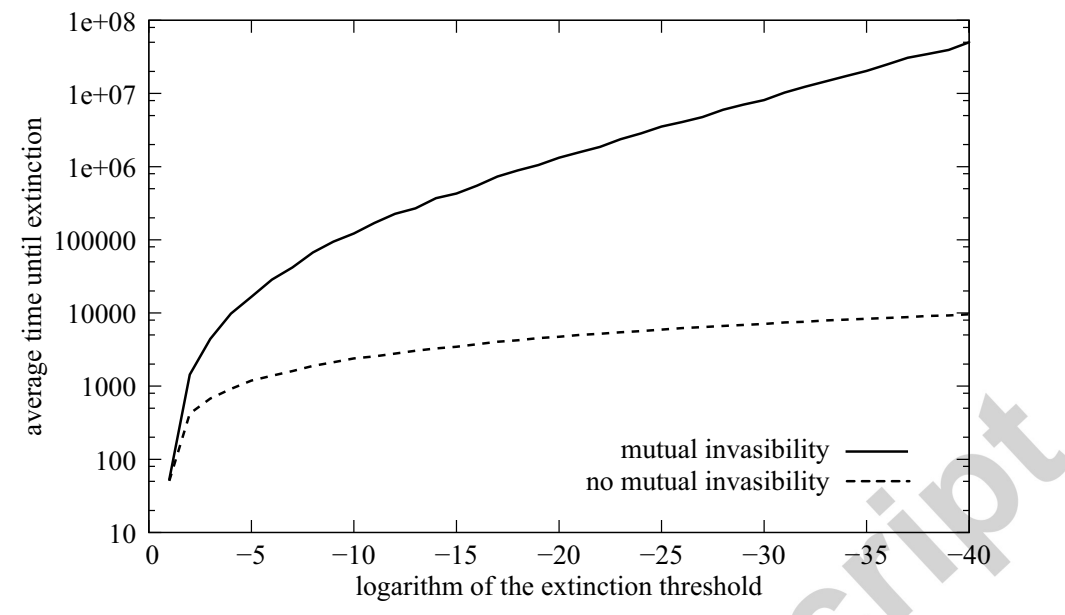

Figure 3: Expected time until extinction as a function of the extinction threshold (simplified model, average of 500 runs). Continuous line: $K_{2}=0.29$ satisfying the condition for mutual invasibility. The coexistence time diverges for low thresholds and becomes inversely proportional to the threshold. Dotted line: $K_{2}=0.19$, no mutual invasibility. Coexistence time remains essentially bounded. 


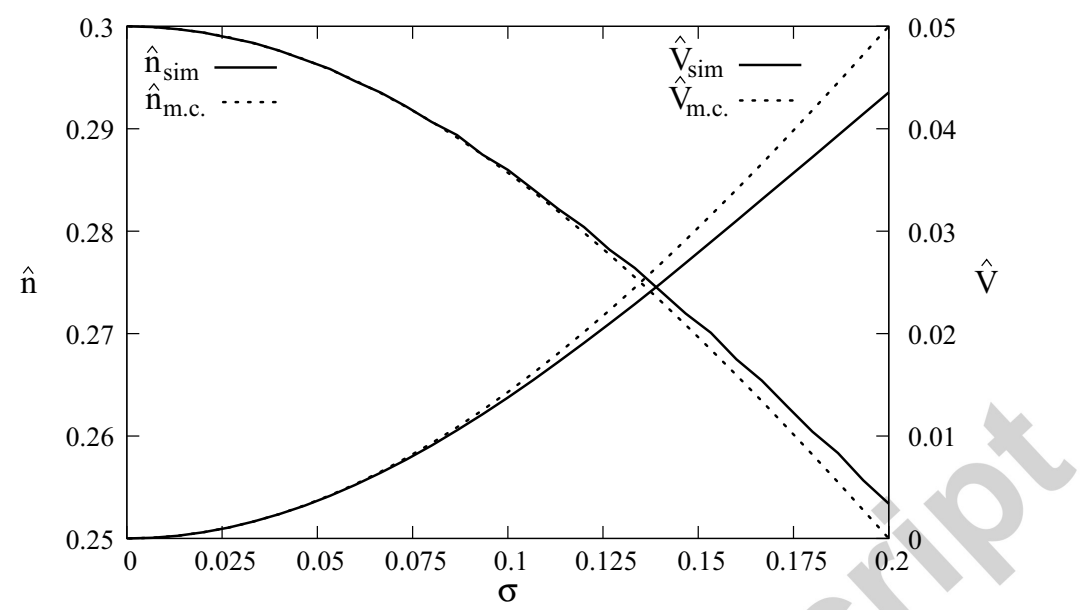

Figure 4: Average density $(\hat{n})$ and variance $(\hat{V})$ of a single population as a function of the fluctuation strength $\sigma$. The moment closure method (dashed line) approximates the simulation results (solid line) very well for $\sigma<0.1$ and reasonably well for $0.1<\sigma<0.2$. Parameters: $a=0.1, b=0.1, K=0.3$. 


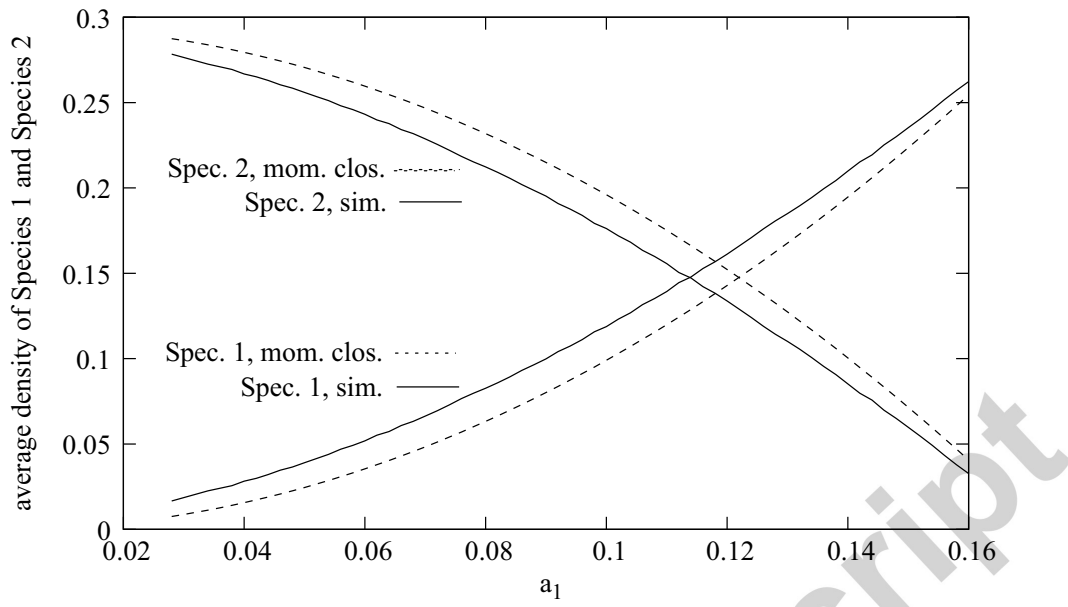

Figure 5: Average densities of two coexisting species as a function of $a_{1}$ in the simplified model. The difference between the moment closure (dashed lines) and the simulated (solid lines) densities are significantly larger than in the single species case of Fig. 4. Still, moment closure remains a reasonable approximation. $K_{2}=0.295$, within the range of mutual invasion. 


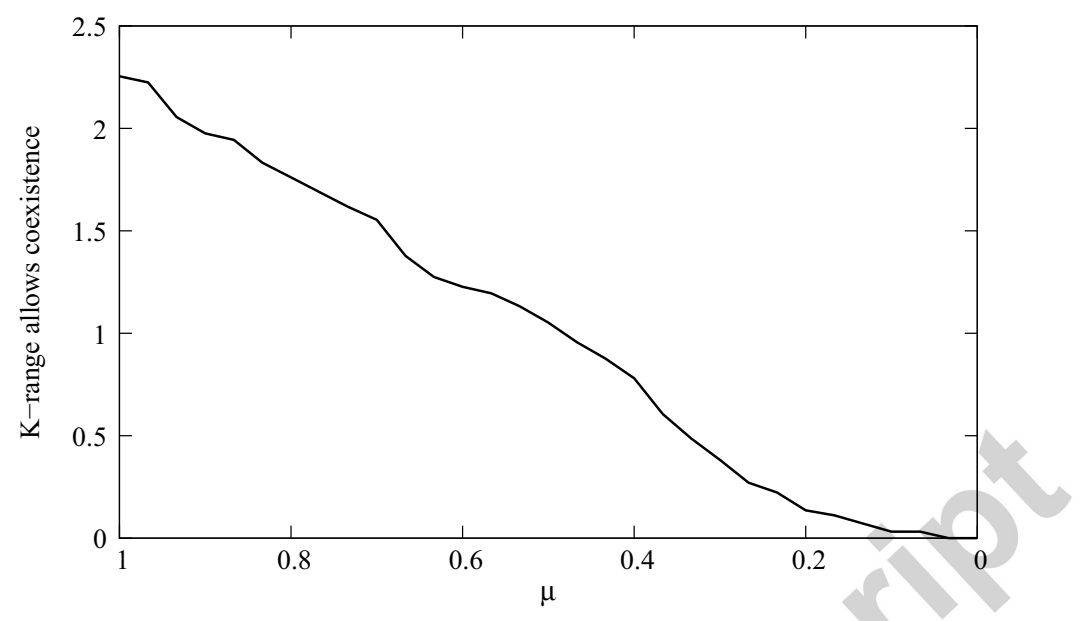

Figure 6: Robustness of coexistence as a function of similarity. The vertical axis is the ratio of the parameter region $\left(K_{1}, K_{2}\right) \in[0,0.3] \times[0,0.3]$ allowing coexistence in arbitrary units. Parameters: $a=0.1 ; b=0.1 ; \sigma=0.1$, the extinction limit $L I M=10^{-40}$. 


\section{Appendix A. Stratonovich and Itô interpretation of stochastic dy- namics}

Caution is needed in defining a "stochastic" integral, like

$$
\int g(t) \xi(t) \mathrm{d} t=\int g(t) \mathrm{d} W(t),
$$

where $\xi(t)$ is the white noise. Here $W(t)$ denotes the Wiener process, the derivative of which is the white noise:

$$
\mathrm{d} W=\xi \mathrm{d} t
$$

Note that

$$
\overline{(\mathrm{d} W)^{2}}=\mathrm{d} t,
$$

a quite nontrivial feature of the white noise.

The integral (A.1) can be approximated by two different sums, referred to as "Stratonovich" and "Itô" types:

$$
\begin{array}{ll}
\text { (S) } & \sum_{l} \frac{g\left(t_{l+1}\right)+g\left(t_{l}\right)}{2} \Delta W\left(t_{l}\right) \\
(\mathcal{I}) & \sum_{l} g\left(t_{l}\right) \Delta W\left(t_{l}\right) .
\end{array}
$$

(Here we use function notation instead of indices to denote time dependence; $\Delta$ means difference between values at $t_{l+1}$ and $t_{l}$.) The difference between the two sums is

$$
(\mathcal{S}-\mathcal{I}) \quad \sum_{l} \frac{1}{2} \Delta g\left(t_{l}\right) \Delta W\left(t_{l}\right) .
$$

Were $g$ and $W$ smooth functions, each term of (A.6) would be proportional to $(\Delta t)^{2}$ and the difference between the two approximations would disappear in the limit $\Delta t \rightarrow 0$. However, because of the property (A.3), if $\Delta g$ is proportional to $\Delta W$, then the expectation of a term is proportional to $\overline{(\Delta W)^{2}}=\Delta t$. Then, the difference $(\mathcal{S}-\mathcal{I})$ remains finite in the limit; the sums $(\mathcal{S})$ and $(\mathcal{I})$ converge to two different values. These two limits constitute two different definitions of the stochastic integral (A.1). For stochastic differential equations (SDE) the two different ways of integration establishes two different solutions, i.e. two inequivalent interpretations of the SDE.

We assume that in the real word the fluctuations are correlated, i.e. the environment cannot change arbitrarily fast. Then, we assume that the correlation time is short compared to the timescale of population dynamical effects. Therefore, we study the limit of zero correlation time, as specified by Eq. (1), leading to Stratonovich-interpretation of Eq. (2) (Braumann 1999, 2007). It is something different than considering the continuous-time process as a limiting case of a discrete dynamics with independent and identically distributed (i.i.d.) random variables as noise, e.g. Itô-interpretation (Feldman and Roughgarden 1975). 
Bored by the growing volume of the Itô-Stratonovich literature, Smythe et al. (1983) established the validity of the Stratonovich approach for describing continuous stochastic phenomena experimentally. They observed also that the naive numerical discretization, corresponding to the Itô-interpretation, leads to an incorrect result.

Consider now the discretization of our model defined by the SDE (2). Naively, one would arrive at the iteration

$$
n_{i, t+\Delta t}-n_{i, t}=\left[-a_{i}\left(n_{t}-K_{i}\right)-b_{i}\left(n_{t}-K_{i}\right)^{2}\right] n_{i, t} \Delta t+\sigma_{i} n_{i, t} \sqrt{\Delta t} \xi_{t} .
$$

(The factor $\sqrt{\Delta t}$ ensures compliance with Eq. (A.3) when the i.i.d. $\xi_{t}$ has a variance 1.) However, the $\Delta t \rightarrow 0$ limit of this iteration would lead to the Itô, instead of the Stratonovich-interpretation of the stochastic dynamics (2). One has to take into account the correction (A.6).

Only the stochastic term of the iteration (A.7) is of interest. Then $\Delta W(t)$ corresponds to $\sqrt{\Delta t} \xi_{t}$ and $g(t)=\sigma_{i} n_{i t}$; therefore $\Delta g\left(t_{i}\right)=\sigma_{i} \Delta n_{i}\left(t_{i}\right)$. However,

$$
\Delta n_{i}\left(t_{i}\right)=\sigma_{i} n_{i}\left(t_{i}\right) \Delta W\left(t_{i}\right)
$$

by the iteration; only the stochastic term was taken into account. Therefore,

$$
\Delta g\left(t_{i}\right)=\sigma_{i}^{2} n_{i}\left(t_{i}\right) \Delta W\left(t_{l}\right)
$$

and a term in the correction (A.6) reads as

$$
\frac{1}{2} \sigma_{i}^{2} n_{i}\left(t_{i}\right)\left(\Delta W\left(t_{l}\right)\right)^{2}=\frac{1}{2} \sigma_{i}^{2} n_{i}\left(t_{i}\right) \Delta t
$$

The expectation value of this correction leads to the correction term $\frac{1}{2} \sigma_{i}^{2} \bar{n}_{i}$ in the iteration (24).

We proceed to prove Eq. (28). Averaging is defined via integration, so the considerations above apply. The Itô average of the product $m \xi$ is zero: value of $m$ in each time step depends only on the values of $\xi$ of the previous steps, which is independent from the current $\xi$. Therefore the Stranovich average $\overline{m \xi}$ comes entirely from the Stratonovich-Itô correction. As now $g(t)$ corresponds to $m(t)$ and $\Delta m=\Delta n$, Eq. (A.8) leads to the relation (28). 